Estudios Fronterizos, vol. 10, núm. 19, enero-junio 2009, pp. 157-181

\title{
Vivienda para los pobres: instrumentación e impactos del programa Tu Casa en la ciudad de Tijuana
}

\author{
Ana Elizabeth Jardón Hernández* \\ Gerardo Ordóñez Barba**
}

Resumen. En el presente estudio se evalúan el funcionamiento y algunos de los principales resultados de uno de los programas federales más importantes en materia de vivienda dirigido a hogares en situación de pobreza, haciendo especial énfasis en su proceso de instrumentación e impactos frente al problema habitacional de las familias pobres en la ciudad de Tijuana. A partir de este trabajo se pretende dar cuenta de los cambios en el diseño del programa, que forzaron su transformación de Vivah a Tu Casa, de las dificultades operativas que enfrenta para implementarse adecuadamente en un contexto urbano determinado y de sus limitados efectos para resolver las necesidades de vivienda de tipo popular. No obstante, también se busca ponderar la importancia de los subsidios gubernamentales para la adquisición o el mejoramiento de viviendas, en su modalidad progresiva, como una alternativa para los segmentos sociales económicamente menos favorecidos y que se encuentran excluidos de cualquier otra opción de financiamiento público o privado.

Palabras clave: pobreza, política habitacional, vivienda progresiva, subsidios, Tijuana.

Abstract. This study assesses the operation and some major results of most important federal housing program focus on households in poverty situation, with particular emphasis on their instrumentation process and impacts in Tijuana's housing problem for poor families. The exam aims to analyses the design changes of the program that forced its transformation from Vivah to Tu Casa, and the operational difficulties for a proper implementation in an urban context, as well as its limited impact for solving popular housing needs. It also seeks to weigh the importance of government subsidies to acquire or improve housing, in their progressive modality, as an alternative for social segments excluded from any other public or private financing option.

Keywords: poverty, housing policy, progressive housing, subsidies, Tijuana.

\footnotetext{
* Maestra en Desarrollo Regional, Departamento de Proyectos Especiales de El Colegio de la Frontera Norte. Correo electrónico: ajardon@colef.mx

** Profesor-investigador del Departamento de Estudios de Administración Pública de El Colegio de la Frontera Norte. Miembro del Sistema Nacional de Investigadores (SNI), nivel II. Correo electrónico: ordonez@colef.mx
} 
ESTUDIOS FRONTERIZOS, VOL. 10, NÚM. 19, ENERO-JUNIO 2009

\section{Introducción}

Con la creación del Fondo Nacional de Habitaciones Populares (Fonhapo) y del Instituto Nacional de la Vivienda en la década de los cincuenta, el Estado mexicano comienza a tener un papel importante en el financiamiento y la construcción de viviendas para familias de escasos recursos, tanto del medio rural como urbano. En las dos décadas siguientes la Federación fue asumiendo mayores compromisos en esta materia, que culminaron con el establecimiento de tres nuevos fondos orientados a cubrir necesidades habitacionales de sectores medios (el Fovi en 1963) y de las clases trabajadoras afiliadas a la seguridad social (el Infonavit y Fovissste en 1972). Hacia mediados de los setenta el sector público en su conjunto ya producía alrededor del $26 \%$ de los inmuebles habitacionales en el país, es decir, 10\% más que los financiados por la banca comercial (Ordóñez, 2002:108-111).

No obstante la amplitud de la cobertura potencial asociada a estos organismos, la mayor parte de los recursos públicos (87\% en 1974) estaba concentrada en los institutos de vivienda que atendían a los sectores medios con capacidad de pago y empleos formales (Ordónez 2002:108-111); como una forma de subsanar parcialmente esta inequidad, al núcleo básico del andamiaje institucional se fueron agregando nuevos programas que buscaban atender de algún modo la problemática habitacional de los hogares marginados o en situación de pobreza (Schteingart, 1988). Con ese propósito se crea en 1980 el Programa de Mejoramiento de la Casa Rural en Zonas Marginadas de la Coplamar, en 1989 inician dos subprogramas integrados al Pronasol (Crédito a la Palabra y Vivienda Digna), y en 1998 se abre el Programa de Ahorro, Subsidio y Crédito para la Vivienda Progresiva Vivah, el cual fue renombrado en 2003 como Tu Casa.

Con la instrumentación de estas iniciativas el gobierno federal también emprendía una estrategia distinta para solucionar los problemas habitacionales de las familias pobres: se pasó de una oferta basada principalmente en la provisión de viviendas terminadas, a "un enfoque de aproximación por etapas", en el que los programas de vivienda progresiva adquirieron un papel central (Coplamar, 1986:79). Las sucesivas crisis económicas de 1982 y 1994 contribuyeron a apuntalar 
esta posición en el diseño de la política habitacional en el país, convirtiéndose junto con la focalización en los enfoques predominantes de las acciones orientadas a la población de escasos recursos.

Desde el punto de vista presupuestal, la adopción de nuevos programas no siempre ha significado mayores recursos; sobre todo a partir de 1994 hemos presenciado una reducción paulatina del financiamiento disponible para los segmentos de población más pobres, que se integran principalmente por hogares cuyo ingreso familiar es inferior a 3 salarios mínimos. Esta tendencia ha contribuido a acentuar las desigualdades sociales en el acceso a una vivienda digna, lo cual resulta contrario al texto constitucional y continúa obligando a millones de mexicanos a vivir en condiciones precarias, insalubres y peligrosas (Villavicencio, 1997).

En este contexto, el presente trabajo tiene como propósito fundamental exponer los resultados de una evaluación aplicada al programa Tu Casa, es decir, al único programa federal existente encargado de atender la problemática habitacional de las familias pobres en el país, tomando como escenario de su intervención a la ciudad de Tijuana en el periodo 2002-2006. Para cumplir con este objetivo, en un primer momento se presentará un breve diagnóstico de las principales necesidades habitacionales que privaban en la ciudad en el año 2000, particularmente de los segmentos de población ubicados bajo la línea de pobreza patrimonial. Esta aproximación nos permitirá posteriormente valorar los alcances y la pertinencia de la iniciativa gubernamental ante el tamaño y las características de la problemática social que se pretende intervenir. En el segundo apartado se analizarán los cambios que experimentó el programa en su diseño cuando transitó de Vivah a Tu Casa; en la tercera parte se hará un recuento de las dificultades de la instrumentación en el contexto de Tijuana, considerando problemas relacionados con la selección de su población objetivo y la ejecución de las obras; por último, se mostrarán algunos de los resultados e impactos más importantes entre sus beneficiarios. Como conclusión, se buscará valorar la eficacia de este tipo de programas y de su enfoque "progresivista", considerando las necesidades de vivienda de una gran parte de la sociedad mexicana en el ámbito urbano. 


\section{Necesidades habitacionales de los hogares en pobreza en Tijuana}

Para analizar las necesidades de vivienda que privan en Tijuana, particularmente de los hogares pobres, se recurrirá a dos tipos de estimaciones que se aplicaron a la muestra del 10\% del XII Censo General de Población y Vivienda de 2000: la primera consistió en una cuantificación de la pobreza tomando como base la metodología oficial establecida por la Secretaría de Desarrollo Social (Sedesol); ${ }^{1}$ el segundo tipo de cálculos se refiere a la cuantificación de dos de las principales problemáticas habitacionales que se viven en el ámbito urbano: la baja calidad en la construcción de pisos, paredes y techos, que se medirá a través de un Índice de Consolidación de la Vivienda (ICV), ${ }^{2}$ y el hacinamiento, para el que se empleará la norma de más dos residentes por dormitorio.

Para complementar la panorámica se utilizarán mediciones realizadas en otro estudio para determinar la demanda anual de vivienda en la ciudad entre los sectores sociales de menores recursos. Desde el punto de vista de las políticas públicas, cada uno de estos aspectos remite a acciones diferenciadas $\mathrm{y}$, según su magnitud, permite valorar la pertinencia y los alcances de los programas orientados al mejoramiento, ampliación o financiación de nuevas viviendas.

\footnotetext{
${ }^{1}$ En este estudio se considera una tipificación que incluye los siguientes tres niveles de pobreza: 1. umbral de pobreza alimentaria: hogares cuyo ingreso por persona era menor al que se consideró como necesario para cubrir las necesidades de alimentación, es decir, de 20.9 pesos diarios de 2000; 2. umbral de desarrollo de capacidades: hogares cuyo ingreso por persona era menor al necesario para cubrir las necesidades de alimentación, educación y salud, lo que equivalía a 24.7 pesos diarios de 2000; y 3. umbral de desarrollo de patrimonio: hogares cuyo ingreso por persona era menor al necesario para cubrir las necesidades de alimentación y el consumo básico en salud, educación, vestido, calzado, vivienda y transporte público, equivalente a 41.8 pesos diarios de 2000 (Sedesol, 2003:2-3).

${ }^{2}$ El Índice de Consolidación de la Vivienda (ICV) se construyó con base en las siguientes tres variables: material predominante en las paredes o muros, techo y piso de las viviendas, con las cuales se conformaron 5 categorías que determinan la calidad constructiva de las viviendas: un ICV muy bajo implica paredes y techo de material de desecho con piso de tierra; bajo, paredes y techo de carrizo, palma, madera con pisos de madera, mosaico o cemento; medio, paredes y techo de lamina de cartón, asbesto y pisos de madera, mosaico o cemento; alto, paredes de madera o adobe, techo de teja y pisos de madera, mosaico o cemento; y muy alto, paredes y techo de tabique, ladrillo y piso de cemento o firme (El Colef, 2005).
} 
A partir de la estimación de la pobreza en Tijuana se encontró que de los 293790 hogares que había en 2000, 105012 se situaban por debajo de la línea de pobreza patrimonial (35.7\%). Cabe mencionar que de acuerdo con estos datos, 188778 son hogares no pobres (es decir, $64.2 \%$ del total) en tanto que contaban con un ingreso per cápita familiar superior a 41.8 pesos diarios.

Es indispensable precisar que en la conceptualización de los umbrales de pobreza cualquier persona o familia que se encuentre por debajo de la línea de desarrollo patrimonial tendrá, por definición, un ingreso insuficiente para satisfacer sus necesidades de vivienda con las características mínimas para proveer protección e higiene a sus habitantes, independientemente que conforme vaya cayendo en los umbrales habrá de padecer mayores carencias, hasta llegar a niveles de indigencia en el caso de quienes se encuentren por debajo de la línea de pobreza alimentaria. Desde este punto de vista, el indicador de pobreza patrimonial ofrece una primera aproximación a las dimensiones de la problemática habitacional de los hogares pobres de Tijuana, lo cual también permite profundizar en las características de sus viviendas.

Respecto de las carencias constructivas, se observa que para el total de viviendas en Tijuana $43.4 \%$ presenta un grado de consolidación

Cuadro 1. Distribución del Índice de Consolidación de las Viviendas de los hogares según su situación de pobreza en Tijuana, 2000 (\%)

\begin{tabular}{lccc}
\hline $\begin{array}{l}\text { Índice de Consolidación } \\
\text { de la Vivienda }\end{array}$ & $\begin{array}{c}\text { Hogares en } \\
\text { pobreza } \\
\text { patrimonial }\end{array}$ & $\begin{array}{c}\text { Hogares } \\
\text { no pobres }\end{array}$ & Total \\
\hline Muy bajo-bajo-medio & 53.4 & 37.9 & 43.4 \\
Alto-muy alto & 46.6 & 62.1 & 56.6 \\
Total & 100.0 & 100.0 & 100.0 \\
Total absoluto & 105,012 & 188,778 & 293,790
\end{tabular}

Fuente: Elaboración propia con base en el XII Censo General de Población y Vivienda 2000. 
(medio, bajo o muy bajo) que implica la presencia de espacios construidos con materiales de desecho o poco durables en techos y paredes, y, en el peor de los casos, con pisos de tierra. En general, estas viviendas no proveen mínimos de seguridad y confort a sus habitantes. Como se aprecia en el cuadro 1, este problema no es exclusivo de la pobreza pues alrededor de $38 \%$ de las familias no pobres habita en viviendas con un grado de consolidación de medio a muy bajo. No obstante, para los hogares en pobreza más de la mitad de sus viviendas (53.4\%) presenta los mismos niveles de consolidación, lo cual pone de manifiesto la magnitud de la problemática asociada a la precariedad en la construcción de los inmuebles habitacionales entre la población de menores recursos en Tijuana. En términos absolutos, los cálculos indican que alrededor de 56 mil hogares pobres en la ciudad residen en viviendas que requieren algún tipo de mejora o sustitución de los materiales con los que fueron construidas.

Desde el punto de vista de las insuficiencias de los espacios utilizados como dormitorios, el indicador de hacinamiento nos muestra que si bien el problema tampoco es privativo de los hogares en pobreza, entre éstos la magnitud del fenómeno casi se triplica respecto de las viviendas de las familias no pobres. En números absolutos, en 2000 había en Tijuana 21552 hogares de escasos recursos que habitaban inmuebles inadecuados al número de sus miembros.

Cuadro 2. Distribución porcentual de los hogares según la situación de hacinamiento que priva en sus viviendas en Tijuana, 2000

\begin{tabular}{lccr}
\hline Hacinamiento & $\begin{array}{c}\text { Hogares en } \\
\text { pobreza } \\
\text { patrimonial }\end{array}$ & $\begin{array}{c}\text { Hogares } \\
\text { no pobres }\end{array}$ & Total \\
\hline Viviendas no hacinadas & 79.5 & 93.0 & 88.2 \\
Viviendas hacinadas & 20.5 & 7.0 & 11.8 \\
Total & 100.0 & 100.0 & 100.0 \\
\hline
\end{tabular}

Fuente: Elaboración propia con base en el XII Censo General de Población y Vivienda 2000. 
Para tener una idea más precisa del tipo de necesidades habitacionales que existen entre las familias pobres en la ciudad, se realizó un procesamiento adicional en el que cruzamos los dos indicadores anteriores, obteniendo los siguientes resultados: 56032 hogares (es decir, 53.4\% de las familias en pobreza) habitan viviendas cuya calidad constructiva es precaria, $21552(20.5 \%)$ residen en viviendas hacinadas y 15047 (14.3\%) ocupan inmuebles con ambos tipos de problemas. Cabe destacar que del total de hogares pobres, un poco más del $40 \%$ vive en viviendas con un alto grado de consolidación y con espacios suficientes de acuerdo con el número de sus miembros. Aún así, el total de estos hogares que sí padecen alguna de estas dos problemáticas ascendió a 62537 en el año 2000.

Por último, según datos de la Comisión Nacional de Fomento a la Vivienda (Conafovi), se estima que la demanda anual de vivienda en Tijuana entre 2000 y 2010 es de 36238 unidades, de las cuales 6857 corresponden a requerimientos de hogares cuyos ingresos son menores a dos salarios mínimos y 8367 para familias que reciben entre 2 y 5 salarios mínimos. En otras palabras, de la demanda anual alrededor de $42 \%$ es requerida por hogares en condiciones económicas poco favorables (Hipotecaria Nacional, 2005). Desde el punto de vista de la oferta, en el 2005 apenas se habían construido 5190 viviendas de interés social y ninguna de tipo económica, lo cual nos indica que el nivel de desatención a la demanda de vivienda nueva alcanza a dos terce-

Cuadro 3. Hogares en pobreza según la situación de consolidación y hacinamiento que priva en sus viviendas en Tijuana, 2000.

\begin{tabular}{lcrr}
\hline Consolidación de la vivienda & $\begin{array}{c}\text { Viviendas } \\
\text { no hacinadas }\end{array}$ & $\begin{array}{l}\text { Viviendas } \\
\text { hacinadas }\end{array}$ & Total \\
\hline Muy bajo-bajo-medio & 40985 & 15047 & 56032 \\
Alto-muy alto & 42475 & 6505 & 48980 \\
Total & 83460 & 21552 & 105012 \\
\hline
\end{tabular}

Fuente: Elaboración propia con base en el XII Censo General de Población y Vivienda, 2000. 
ras partes $(66 \%)$ de hogares con ingresos inferiores a 5 salarios mínimos y al 100\% de aquellos que reciben menos de 2 .

En conjunto, los datos anteriores ofrecen una aproximación a algunas dimensiones de la problemática habitacional que enfrenta, en una ciudad fronteriza del norte de México, el único programa federal diseñado para atender las necesidades de las familias pobres. Estamos conscientes de que este breve diagnóstico no reconstruye la complejidad asociada al problema habitacional; por razones de espacio no hemos querido abundar sobre otros aspectos importantes, como lo son la tenencia de la tierra, el acceso a servicios básicos, el estado del entorno urbano, entre otros. Sin embargo, lo ya establecido nos permitirá contar con elementos para valorar los alcances y la pertinencia de las tres estrategias centrales de Tu Casa: mejoramiento, ampliación y adquisición o edificación de viviendas nuevas. Para iniciar este abordaje, enseguida habremos de dar cuenta de las características de esta

Cuadro 4. Demanda y oferta de vivienda según el ingreso en el hogar en Tijuana

\begin{tabular}{|c|c|c|c|}
\hline \multicolumn{2}{|c|}{$\begin{array}{l}\text { Demanda anual según niveles de } \\
\text { ingreso en el hogar y tipo de vivienda, } \\
\text { 2000-2010 }\end{array}$} & \multicolumn{2}{|c|}{$\begin{array}{l}\text { Oferta según tipo de vivienda } \\
\text { y segmentos de valor, } 2005\end{array}$} \\
\hline $\begin{array}{l}\text { Menos de } 2 \text { veces el salario } \\
\text { mínimo (VSM) / Vivienda } \\
\text { económica }\end{array}$ & 6,857 & $\begin{array}{l}\text { Vivienda económica (con un } \\
\text { valor menor a } 210 \text { mil pesos) }\end{array}$ & 0 \\
\hline $\begin{array}{l}\text { Más de } 2 \text { y menos de } 5 \text { VSM / } \\
\text { Vivienda económica, social e } \\
\text { interés medio }\end{array}$ & 8,367 & $\begin{array}{l}\text { Vivienda social (entre } 210 \mathrm{mil} \\
\text { y } 300 \text { mil pesos) }\end{array}$ & 5190 \\
\hline $\begin{array}{l}\text { Más de } 5 \text { y menos de } 12 \text { vSM / } \\
\text { Vivienda social, interés medio } \\
\text { y media }\end{array}$ & 14,577 & $\begin{array}{l}\text { Vivienda de interés medio } \\
\text { (entre } 300 \text { mil y } 500 \text { mil pesos) }\end{array}$ & 1272 \\
\hline \multirow[t]{2}{*}{$\begin{array}{l}\text { Más de } 12 \text { VSM / Vivienda } \\
\text { media, residencial y residencial plus }\end{array}$} & s, 637 & $\begin{array}{l}\text { Vivienda media (entre } 500 \text { mil y } \\
900 \text { mil pesos) }\end{array}$ & 475 \\
\hline & & $\begin{array}{l}\text { Vivienda residencial (más de } \\
900 \text { mil pesos) }\end{array}$ & 598 \\
\hline Totales & 36238 & & 7535 \\
\hline
\end{tabular}

Fuente: Elaboración propia con datos de Hipotecaria Nacional, 2005. 
iniciativa y las transformaciones que experimentó entre 2003 y 2006 a raíz del cambio en su denominación.

\section{De Vivah a Tu Casa: la evolución en el diseño del programa, 2002-2006}

Con el propósito de homologar los lineamientos de los programas de vivienda y reducir sus costos de operación, el Programa Sectorial de Vivienda 2001-2006 consolidó al Fideicomiso del Fondo Nacional de Habitaciones Populares (Fonhapo) como el organismo encargado de operar los recursos económicos de los programas federales de fomento a la vivienda para las familias de escasos recursos (Fonhapo, 2004:1-8).

Así, en el convenio suscrito el 18 de junio de 2002 entre la Sedesol y el Fonhapo se establecen las bases para que este último participe en la operación del programa Vivah, que había sido creado en 1998 durante el gobierno de Ernesto Zedillo. A partir de entonces se introducen una serie de modificaciones en sus alcances y denominación social, pues para 2003 adquiere el nombre de Programa de Ahorro, Subsidio y Crédito para la Vivienda Progresiva Tu Casa (Fonhapo, 2003:1-29).

Uno de los aspectos principales que muestran una evolución positiva en el diseño del programa se encuentra en la ampliación de las acciones que desarrollaba, ya que antes de que el fideicomiso asumiera la responsabilidad de su operación únicamente promovía la asignación de subsidios por un monto máximo de 10 mil pesos por mejoramiento de vivienda, para las familias con predios regulares que construyeron habitaciones con materiales endebles. Para 2003, además de considerar la modalidad de mejoramiento habitacional, se incorporan las de ampliación, adquisición y edificación de una Unidad Básica de Vivienda (UBV) en terreno propiedad del gobierno o del beneficiario, mientras que para 2004 se diferencia según modalidad y dimensión territorial, pues las vertientes de mejoramiento y edificación de una UBV se subdividen en vivienda urbana y rural.

Por último, en 2006 se establece como una nueva modalidad de apoyo la adquisición de una Unidad de Vivienda Económica (UVE) para quienes cuentan con crédito aprobado por organismos naciona- 
les o estatales, y se conserva la UBV para los beneficiarios calificados por el Sistema Integral de Información del Fonhapo. Además, en este mismo año se incorpora la modalidad de adquisición de un lote con servicios.

En términos generales, la ampliación en los alcances del programa se sintetiza en su objetivo central: "Mejorar las condiciones de vida de la población que vive en pobreza patrimonial, mediante el otorgamiento de un subsidio federal para adquirir, edificar, ampliar o mejorar sus vivienda o adquirir un lote con servicios".

Es conveniente señalar la complejidad que implica el esquema de operación del programa, pues su diseño exige de la coordinación institucional entre cuatro instancias que teóricamente participan en su implantación: i) instancia normativa, ii) instancia ejecutora, iii) instancia corresponsable, y iv) instancias auxiliares de la instancia normativa; en concreto, para su correcta operación Tu Casa requiere de la participación conjunta del Fonhapo (i), de los gobiernos estatales o municipales (ii), según sea el caso, de las delegaciones federales de la Sedesol (iii), y de los Fondos u Organismos Nacionales de Vivienda (Onavi's), así como de otras instituciones que determine el propio fideicomiso (iv).

Este esquema de coordinación pretende dar fortaleza a la asignación de los fondos federales, pues en el compromiso que adquieren los estados y municipios que celebran convenio con el fideicomiso, se establece que los subsidios otorgados a los beneficiarios deberán ser en igual cantidad a los aportados por la Federación. En donde según sea el caso, la contribución del beneficiario se concreta en la aportación del ahorro que le corresponde, completando así la fórmula del esquema que combina la asignación del subsidio federal + subsidio estatal + ahorro familiar. Un esquema complejo que busca incrementar significativamente los apoyos a la población en pobreza, pero que también puede no materializarse por la ausencia de alguno de estos elementos.

En la evolución del programa, los montos aportados por los actores involucrados observan un ligero incremento, siendo el más importante el que se presenta una vez que el fideicomiso se responsabiliza de su operación y se amplían las modalidades de intervención. En su diseño, las acciones de vivienda promovidas resultan congruentes con la situación de pobreza de muchos de los hogares en México, pues 


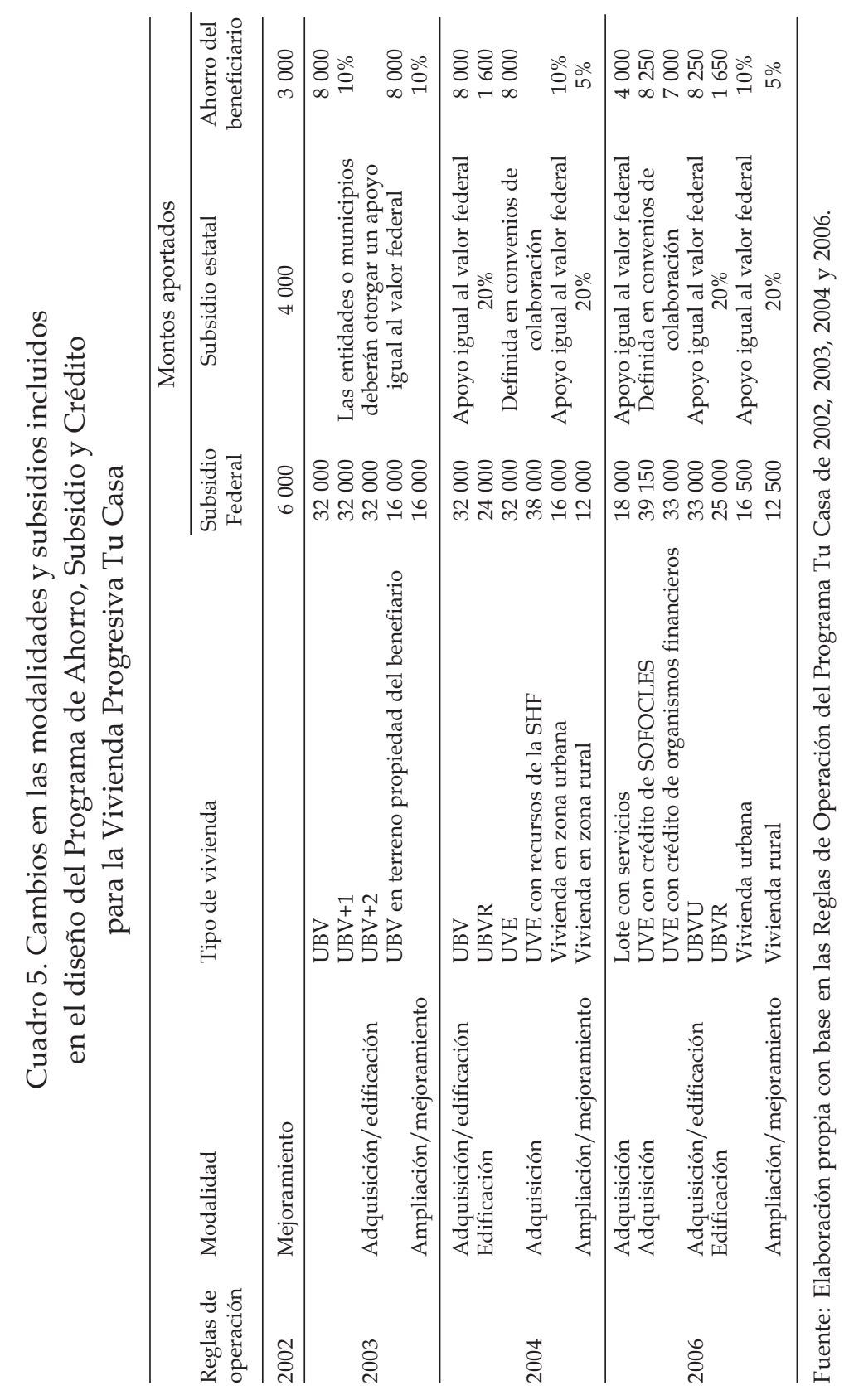


la población de reducida capacidad adquisitiva, con un ingreso apenas suficiente para sobrevivir, difícilmente puede adquirir una vivienda nueva bajo los esquemas crediticios con los que actualmente trabajan las Sociedades Financieras de Objeto Limitado (Sofoles) y las instituciones bancarias en el país. En una primera apreciación se podría decir que Tu Casa representa una alternativa de solución al problema de la vivienda popular, al fomentar acciones de financiamiento a la vivienda progresiva y mejorada.

\section{La implantación del programa Tu Casa en la ciudad de Tijuana}

En la coordinación para la implantación de Tu Casa en Tijuana durante el periodo que cubre este trabajo, estuvieron involucrados, además del Fonhapo como entidad normativa, la delegación de la Sedesol en Baja California como instancia de control y vigilancia con facultades para dar seguimiento a las acciones desarrolladas en el municipio, y la Inmobiliaria Estatal de Tijuana-Tecate (Inett) -de aquí en adelante la Inmobiliaria- como entidad ejecutora responsable de validar las Cédulas de Información Socioeconómica (CIS) que se aplican a posibles beneficiarios, coordinar los procesos de construcción y aportar los terrenos urbanizados, entre otras atribuciones estipuladas en las Reglas de Operación del Programa (ROP).

Aun cuando el objetivo de este trabajo no es analizar los problemas que puedan resultar de la coordinación institucional, es conveniente señalar la complejidad del esquema propuesto para la operación del programa, sobre todo por las competencias que adquieren los estados y municipios involucrados. En suma, la gestión intergubernamental desempeña un papel importante para garantizar la eficacia de sus resultados. No obstante, habremos de valorar dos aspectos que buscan medir el desempeño del programa en su etapa de instrumentación: la selección de beneficiarios y la ejecución de las obras.

La selección de los beneficiarios

Para evaluar el cumplimiento de las ROP en cuanto a uno de los criterios establecidos para la selección de los beneficiarios, se calculó el 
nivel de pobreza de los hogares siguiendo nuevamente la metodología establecida por la Sedesol. Lo anterior con base en las siguientes dos fuentes de información: i) las Cédulas de Información Socioeconómica (CIS) del padrón de beneficiarios del Programa Tu Casa en Tijuana durante los años 2002 y 2003, ${ }^{3}$ y ii) los resultados de los cuestionarios aplicados en 2004 a una muestra aleatoria de dicho padrón. ${ }^{4}$

Con base en la información proporcionada por la Inmobiliaria, durante los años 2002 y 2003, el Fonhapo validó 403 subsidios para familias de escasos recursos de la ciudad de Tijuana: 319 para ampliación y 84 para edificación de vivienda, de los cuales para mayo de 2004, 104 habían sido cancelados ${ }^{5}$ y de ellos 56 fueron sustituidos. Por lo cual, se realizó un análisis de 337 casos, que resultan de restar al universo original 48 subsidios no reasignados y 18 expedientes no disponibles en los archivos de la Inmobiliaria.

\footnotetext{
${ }^{3}$ Las CIS proporcionadas por la Inmobiliara se tomaron de los expedientes integrados para los beneficiarios del programa.

${ }_{4}^{4}$ Con base en los expedientes del padrón de beneficiarios 2002-2003 integrado por la INETT, se estimó una muestra considerando los 403 casos registrados, la cual tuvo un nivel de confianza de $90 \%$ y un error de estimación del $10 \%$. La muestra resultó en un total de 58 cuestionarios, cuya selección se realizó con base en el cálculo de números aleatorios. Los cuestionarios se diseñaron de acuerdo con las modalidades contempladas en las Reglas de Operación de 2003, esto es una cédula para los Jefes de Hogar calificados en el subprograma Crecemos Tu Casa y otra para el subprograma Iniciamos Tu Casa. El cuestionario considera también una cédula general para conocer las características socioeconómicas, la situación de la vivienda, de las impresiones y conocimiento del programa; para identificar algunas debilidades en el proceso de implantación se diseñaron cédulas para acciones pendientes y subsidios cancelados, que de acuerdo al total de cuestionarios aplicados resultaron en 20 registros para obras de ampliación terminadas, 13 acciones pendientes, 11 subsidios cancelados y 11 subsidios para adquisición de una Unidad Básica de Vivienda.

${ }^{5}$ La cancelación de los subsidios puede ser resultado de: i) mora en el pago de las mensualidades que el beneficiario debe pagar como ahorro; ii) renuncia voluntaria; y iii) predios no aptos para la construcción del cuarto en la vivienda. En el primer factor, el subsidio es cancelado siempre que el beneficiario no haya efectuado un pago adicional al enganche con el que cotizó su solicitud de ingreso al programa. El ahorro, o bien la cantidad que el beneficiario debe aportar, es de 3 mil pesos, misma que según su nivel de ingreso se define con un enganche que varía entre 50, 100 o 250 pesos y mensualidades de 245,250 o 263 pesos a pagar durante un año. En el segundo factor, la cancelación del subsidio responde a la renuncia voluntaria de los beneficiarios, y finalmente, un subsidio que es cancela-
} 
Cuadro 6. Distribución porcentual según nivel de pobreza de los hogares beneficiarios del programa Tu Casa en Tijuana

\begin{tabular}{lcc}
\hline Condición de pobreza & $\begin{array}{c}\text { Solicitantes } \\
\text { validados: con } \\
\text { subsidio aprobado } \\
\text { 2002-2003 }\end{array}$ & $\begin{array}{c}\text { Beneficiarios: con } \\
\text { subsidio asignado }\end{array}$ \\
\hline Pobreza alimentaria & 51.9 & 2004 \\
Pobreza de capacidades & 64.4 & 34.5 \\
Pobreza patrimonial & 82.5 & 48.3 \\
No pobres & 8.6 & 75.9 \\
\hline
\end{tabular}

Fuentes: a. Elaboración propia con base en las CIS del padrón de beneficiarios del programa en la ciudad. En $8.9 \%$ de estos registros no se tiene información sobre los ingresos del hogar.

b. Investigación de campo realizada entre mayo y junio de 2004 .

Siguiendo la metodología de la Sedesol para clasificar a los hogares según su nivel de pobreza, se observa que cuando fueron aprobados los subsidios para los beneficiarios del programa, 278 hogares se encontraban por debajo de la línea de pobreza patrimonial y sólo en 29 de ellos $(8.6 \%)$ se percibía un ingreso per cápita diario superior a 46.5 o 48.8 pesos, según el año en que ingresaron su solicitud al programa. ${ }^{6}$ En esta etapa, los resultados muestran que las instancias responsables tuvieron un bajo nivel de error, al otorgar la mayor parte de los subsidios a hogares en pobreza, particularmente a aquellos que se encontraban en una situación de pobreza extrema o alimentaria $(51.9 \%)$.

do porque el terreno no cuenta con las condiciones adecuadas para el proceso de construcción tiene que ver con los materiales utilizados, en tanto que son paneles de concreto premezclados que para su armado o ensamblaje requieren de espacio suficiente para que la grúa que los transporta realice los movimientos necesarios.

${ }^{6}$ Para actualizar los rangos de ingreso per cápita de los hogares en pobreza se consideró el Índice Nacional de Precios al Consumidor Base segunda quincena de junio 2002=100, para los años 2002, 2003 y 2004. De esta manera, para 2002, el ingreso per cápita diario para los hogares en pobreza alimentaria se estimó en 23.2 pesos, en pobreza de capacidades de 27.5 pesos y en pobreza de patrimonio de 46.5 pesos. Para 2003 los valores quedaron en 24.4 , 28.8 y 48.8 pesos diarios, respectivamente. Y para 2004, en 25.5, 29.3 y 51.1 pesos diarios, en el mismo orden. 
Comparando los resultados de las Cédulas de Información Socioeconómica con lo obtenido en trabajo de campo en 2004, se observan cambios importantes en los ingresos de los beneficiarios, pues el porcentaje de hogares no pobres pasa de 8.6 a $24.1 \%$, e igualmente disminuyen las familias en pobreza alimentaria en alrededor de 18 puntos entre un año (2002 o 2003) y otro (2004). Esta situación podría tener varias explicaciones: $i$ ) un incremento en las percepciones salariales de los hogares por ascensos en el empleo, cambios de ocupación o por incorporación del algún miembro al mercado de trabajo; ii) una segunda posibilidad estribaría en efectos positivos asociados a la estabilidad macroeconómica y al aumento de los salarios reales; por último, iii) cabría la eventualidad de una incorrecta verificación y validación de la situación socioeconómica de los beneficiarios al momento de la aplicación de las CIS. Independientemente del origen de estas divergencias, en el peor de los escenarios observamos un desempeño aceptable del organismo ejecutor en la selección de la población objetivo.

La ejecución de las obras

Conforme a lo estipulado en las Reglas de Operación del Programa de 2003, el monto del subsidio asignado en la modalidad ampliación de la vivienda fue de 15 mil pesos, dividido en partes iguales por la Federación y el estado; así como una aportación efectuada por los beneficiarios (3 mil pesos) para complementar el costo total de la obra: de 18 mil pesos. Conviene mencionar que los subsidios aprobados en esta modalidad se destinaron a la construcción de un cuarto de $9 \mathrm{~m}^{2}$ y se distribuyeron en 53 colonias del municipio de Tijuana, entre las que destacan: Cumbres I y II, Nuevo Milenio, Leandro Valle, Sánchez Taboada, Altiplano, Lomas del Valle, Camino Verde y Vista del Valle.

Con base en información obtenida del padrón de beneficiarios integrado por el Departamento de Regularización y Área Social de la Inmobiliaria, en mayo de 2004, aproximadamente dos años después de haberse celebrado el convenio para las obras de ampliación, se tenían únicamente registros para la construcción de 215 habitaciones de $3 \times 3 \mathrm{~m}^{2}$, de las cuales 121 se encontraban pendientes y 94 ya se habían realizado. Estos datos muestran un avance de apenas $29.4 \%$ respecto 
de la meta inicial, en la que se planteó la construcción de 319 cuartosdormitorio; aun descontando los subsidios cancelados, el logro de la meta (acciones terminadas) no supera el $44 \%$.

Cabe mencionar que la información proporcionada por la Inmobiliaria no es del todo consistente con el dictamen de la Comisión de Fiscalización del Gasto Público, que expone la evaluación de la Cuenta Pública Anual de dicha institución para el ejercicio fiscal de 2002.

En este dictamen se estipula que el 24 de octubre de 2002 la Inmobiliaria solicitó a la Secretaría de Infraestructura y Desarrollo Urbano (SIDUE) avalar los trabajos para la construcción de 488 cuartos adicionales de $9 \mathrm{~m}^{2}$ para la ampliación de viviendas en Tijuana, con un periodo de ejecución del 4 de noviembre al 15 de diciembre de 2002. En la solicitud se proponía la aplicación de un método de construcción alternativo a base de paneles prefabricados, el cual hipotéticamente constituía un ahorro económico y menor tiempo de ejecución que el requerido por el método tradicional a base de muros de block. No obstante, se encontró que al cierre del ejercicio de 2002 no existían avances físicos.

Así pues, mediante diversas Actas de Entrega-Recepción "parciales" se avaló la entrega de 273 cuartos en mayo de 2004 y 215 adicionales en agosto del mismo año, completándose entonces la obra pública de construcción de los 488 cuartos adicionales de $9 \mathrm{~m}^{2}$, es decir, más de 660 días naturales posteriores del inicio de las obras convenidas en noviembre de 2002.

Con base en esta información obtenida del dictamen de la Comisión de Fiscalización del Gasto Público, se observan diversas irregularidades en el proceso de ejecución de la obra pública mencionada, que tienen que ver con el incumplimiento en los tiempos de ejecución programados, declaración de finiquito de las obras cuando éstas aún continuaban en proceso de construcción, omisión en el presupuesto autorizado y ejercicio indebido de cantidades para la adquisición de reservas territoriales no suscritas en el convenio respectivo.

Por otra parte, se aprecian también ligeras inconsistencias en el total contemplado en el padrón de beneficiarios, según el cual de 2003 a 2005 un total de 493 hogares recibieron subsidio para vivienda: 403 en 
2003, 42 en 2004 y 48 en 2005. ${ }^{7}$ Lo cual probablemente significa que la suma de los beneficiarios para los tres años se refiere al convenio suscrito originalmente en 2002 para la construcción de los 488 cuartos.

En general, con independencia de los impactos en las condiciones de habitabilidad de los hogares beneficiados, se observan graves problemas en la operación del programa que redundaron en un alargamiento injustificado en los tiempos de entrega de las obras, así como en diversas complicaciones que muy probablemente impidieron una ampliación significativa de sus beneficios a un mayor número de familias en Tijuana. En 2006 el número de beneficiarios de Tu Casa apenas fue de 199 y en 2007 repuntó a 594; si bien en este último año hubo un aumento importante respecto de los tres años anteriores, el número sigue siendo insignificante ante las dimensiones de las necesidades habitacionales de los hogares pobres en la ciudad.

\section{Los impactos del programa Tu Casa en Tijuana}

Antes de iniciar con los resultados de los cuestionarios aplicados a los beneficiarios, cabrían algunas observaciones generales sobre la pertinencia y los alcances del programa ante la problemática habitacional que se padece en Tijuana. Como ya se ha mencionado, considerando las principales necesidades de vivienda existentes entre las familias pobres de la ciudad, la oferta presentada en el diseño de Tu Casa resulta adecuada, en la medida en que promueve, basadas en un enfoque "progresivista", acciones de mejoramiento, ampliación y edificación o adquisición de nuevas viviendas. No obstante, como hemos podido apreciar en el apartado anterior, en nuestro caso de estudio la entidad ejecutora sólo convino con el Fonhapo la realización de acciones en dos modalidades, haciendo a un lado la posibilidad de apoyar la introducción de mejoras en la calidad constructiva de las viviendas, lo que afecta directamente a casi 41 mil familias que viven en inmuebles precarios.

Desde el punto de vista cuantitativo, es notable que en tres años las acciones del programa hayan beneficiado, en el mejor de los casos, a

${ }^{7}$ Para acceder al padrón de beneficiarios véase http://www.fonhapo.gob.mx 
apenas 493 hogares, incluyendo a 84 que pudieron obtener un pie de casa. ${ }^{8}$ En términos relativos, estos apoyos sólo alcanzaron para beneficiar a $1.9 \%$ de los hogares con viviendas hacinadas y a $1.2 \%$ de familias cuyo ingreso total no supera los 2 salarios mínimos y que necesitan de una vivienda nueva en tan sólo un año. En principio, estos datos demuestran un alto grado de desatención ante las dimensiones de las necesidades habitacionales de los hogares pobres de esta ciudad.

Ahora bien, para conocer los principales impactos del programa desde el punto de vista de los que sí fueron apoyados, se aplicó un conjunto de cuatro cuestionarios a una muestra aleatoria del padrón de beneficiarios registrados por la Inmobiliaria. Así, para aquellos cuya obra consistió en la ampliación de la vivienda se rescataron algunos elementos que tienen que ver con la necesidad habitacional de la familia cuando se solicitó el subsidio, características y calidad del cuarto construido e impresiones acerca de los beneficios que resultaron de dicha obra.

Con base en los resultados de esta investigación, se encontró que para aquellos hogares en donde la obra de ampliación ya había sido realizada, 75\% manifestó que necesitaba un dormitorio, $15 \%$ un espacio para cocina y $10 \%$ un baño. En este orden de ideas gran parte de ellos plantea que la construcción del cuarto -aunque de dimensiones pequeñas- satisfizo la necesidad de contar con un espacio adicional habitable.

En palabras de una encuestada, los beneficios recibidos por el Programa se expresan en la sustitución del material de uno de sus dos cuartos de madera, a la vez que mencionó lo siguiente: “...me ayudó, porque si yo tuviera que construirlo con mis propios medios no lo hubiese hecho, en cambio el programa de los cuartitos me ayudó mucho para que me construyeran con tanta facilidad de pago". ${ }^{\prime}$

\footnotetext{
${ }^{8}$ Estas unidades fueron asignadas a través del subprograma Iniciamos Tu Casa que comenzó a operar en la ciudad de Tijuana en mayo del 2003. Las primeras construcciones de este tipo se desarrollaron en tres colonias de la ciudad, distribuidas de la siguiente manera: 58 en Nuevo Milenio, 25 en Lagunitas y una en Alfredo Ames.

${ }^{9}$ Estas impresiones se rescataron de las preguntas abiertas vertidas en los cuestionarios que les fueron aplicados a los beneficiarios del programa.
} 
Con el objetivo de agrupar las impresiones de los beneficiarios respecto de los impactos de las obras de ampliación, se creó un concentrado de opciones que abordan las cuestiones de la situación habitacional y condiciones de vida, en donde en términos generales se observa que gran parte de los beneficiarios tienen percepciones positivas, sobre todo en lo que tiene que ver con el mejoramiento en la calidad y comodidad de su vivienda.

Cabe mencionar que los comentarios de los beneficiarios que expresaron no haber mejorado la calidad de su vivienda, tienen que ver con el tipo de material de construcción, pues los paneles de concreto premezclados hechos a base de unicel ${ }^{10} \mathrm{y}$ cemento presentan dos problemas principalmente: agrietamientos y traspaso de agua en época de lluvias. Sobre éste último aspecto, es importarte aclarar que de acuerdo al "acta de entrega física", la impermeabilización de los cuartos es responsabilidad de los beneficiarios; con ello no se busca exculpar a la Instancia Ejecutora de tales deficiencias, pero sí hacer notar la dificultad de los hogares para realizar los acabados de la obra, entre ellos: impermeabilización, sellado y pintura de muros exteriores, pintura de muros interiores y barnizado de los elementos de madera.

\section{Cuadro 7. Impactos expresados por los beneficiarios del programa Tu Casa con obras de ampliación en la vivienda, 2004}

\begin{tabular}{lcc}
\hline Impactos & Sí & No \\
\hline Redujo el hacinamiento & 75.0 & 25.0 \\
Dejó de utilizar la cocina como habitación & 55.0 & 45.0 \\
Tiene un cuarto independiente & 55.0 & 40.0 \\
Mejoró la calidad de su vivienda & 89.4 & 10.6 \\
Su vivienda es más amplia & 89.4 & 10.6 \\
Su vivienda tiene más valor & 68.4 & 31.6 \\
La familia vive más cómoda & 89.4 & 10.6 \\
\hline
\end{tabular}

Fuente: Elaboración propia con base en investigación de campo, junio de 2004.

\footnotetext{
${ }^{10}$ El unicel o hielo seco se utiliza para contener el colado de los paneles de concreto premezclados.
} 
Con independencia de dichos problemas, resulta interesante que la totalidad de los encuestados mencionó estar satisfecha con los beneficios que obtuvo del programa por tres aspectos principalmente: el primero, la asignación de un subsidio de más de $80 \%$ del costo de la obra; el segundo, las facilidades otorgadas por la Inmobiliaria para finiquitar el ahorro que le corresponde; y el tercero, la oportunidad de contar con un cuarto de materiales duraderos.

No obstante lo anterior, se han identificado también algunas debilidades que no han sido atendidas satisfactoriamente mediante la ejecución de estas obras, entre ellas el porcentaje de hogares con beneficiarios encuestados para los que no fue posible dejar de utilizar la cocina como dormitorio, así como el problema que representa que algunos de los miembros del hogar no cuenten con habitaciones independientes. Otras inconformidades encontradas en el estudio se refieren a: i) la superficie construida de los cuartos, y ii) el material de construcción de los mismos, pues desde la perspectiva de los beneficiarios queda abierta la sugerencia de construir mediante el proceso tradicional a base de block y cemento.

Respecto de las acciones que hasta mayo de 2004 se encontraban pendientes, se tiene que en general son factores internos (complejidad administrativa) y externos (terrenos no aptos o asentamientos en zonas de alto riesgo) a la Inmobiliaria los que influyeron en el corto avance de la meta planteada inicialmente, lo cual pone de relieve un inadecuado control por parte de la Instancia ejecutora en la validación de los subsidios, pues gran parte de las cancelaciones fueron resultado de la presencia de terrenos en condiciones poco adecuadas para realizar las obras según el método de construcción acordado. Situación que teóricamente no debió presentarse, pues entre las atribuciones de la Inmobiliaria se encontraba la verificación de la información proporcionada por los solicitantes. Al no llevarse a cabo dicho procedimiento, se incrementaron notablemente los tiempos de ejecución y esto incidió en la duplicidad de gastos por la cancelación y sustitución de los subsidios aprobados.

En lo que respecta a las obras de edificación y adquisición de Unidades Básicas de Vivienda, la Inmobiliaria impulsó un proyecto para el desarrollo de 84 unidades, que están integradas por un cuarto de 
usos múltiples y un baño en un lote de $160 \mathrm{~m}^{2}$ con posibilidades de ampliación de una y hasta tres recámaras. Todas bajo el mismo esquema de construcción mencionado con anterioridad.

El impacto en la situación habitacional de las familias que recibieron dichas unidades varía en buena medida, pues se trata de hogares que fueron reubicados por encontrarse en zonas de alto riesgo. No obstante, una proporción importante mencionó que ahora cuenta con un patrimonio familiar $(81.8 \%)$, en tanto que en otros casos se manifestó la oportunidad de haber logrado reemplazar su vivienda que estaba construida con materiales ligeros (54.5\%).

En general, alrededor de $80 \%$ de los beneficiarios que recibieron una UBV se muestra satisfecho con el subsidio y la calidad de la vivienda que obtuvieron, esto aunado a las facilidades de pago y la seguridad que les brinda el hecho de vivir en un terreno geográficamente no accidentado. Sin embargo, los mismos entrevistados destacan algunos aspectos negativos asociados con la superficie construida de la vivienda, la fragilidad de los materiales de construcción y la falta de drenaje conectado a la red pública, pues las UBV se entregan con descarga hacia una fosa séptica.

Un aspecto que merece especial atención es el alto porcentaje de beneficiarios $(72.7 \%)$ que manifestó las reducidas posibilidades de ahorrar luego de haber adquirido la UBV, lo cual está estrechamente

Cuadro 8. Principales impactos expresados por los beneficiarios del programa Tu Casa que adquirieron una UBV, 2004

\begin{tabular}{lll}
\hline ¿Con la UBV que adquirió usted piensa que: & Sí & No \\
\hline Sustituyó una vivienda de materiales perecederos? & 54.5 & 45.4 \\
Obtuvo una vivienda propia? & 18.1 & 81.8 \\
Ahora tiene un patrimonio familiar? & 81.8 & 18.1 \\
Mejoró su calidad de vida? & 45.4 & 54.5 \\
Dejó de pagar renta? & 18.1 & 81.8 \\
Le es posible ahorrar? & 27.2 & 72.7 \\
Gasta más en el mantenimiento de la UBV? & 81.8 & 18.1 \\
Respondió a su necesidad habitacional? & 63.6 & 36.3 \\
\hline
\end{tabular}

Fuente: Elaboración propia con base en investigación de campo, junio de 2004. 
relacionado con el hecho de que $81.8 \%$ manifestó utilizar parte importante de sus ingresos en gastos de mantenimiento, entre los cuales destaca el que tiene que ver con el desagüe de la fosa séptica, que de acuerdo con uno de los beneficiarios implica un costo de 300 pesos mensuales.

Así también resulta relevante que la mayor proporción de estas familias (54.5\%) mencionó que la adquisición de la UBV no necesariamente mejoró su calidad de vida, lo cual de acuerdo con las impresiones rescatadas en la investigación tiene mucho que ver con el sentimiento de haber perdido lo que ellos consideraban parte de su patrimonio familiar, pues es importante notar que cerca de la mitad (45.4\%) de estos hogares contaba con una vivienda construida con materiales duraderos (cuadro 8).

Por último, se indagó acerca de la importancia que tiene la construcción progresiva de la vivienda para mejorar la situación habitacional de los hogares en pobreza; así, desde el punto de vista de los beneficiarios entrevistados, se encontró que 90.9\% la considera como la mejor opción, dada la falta de recursos para obtener una vivienda con ingresos apenas suficientes para sobrevivir, la dificultad para construir de manera inmediata, pero sobre todo porque al adquirir una UBV obtienen seguridad jurídica sobre su terreno.

\section{Consideraciones finales}

No hay duda de que, de acuerdo con el diagnóstico de la problemática habitacional que padecen los segmentos de población con ingresos más bajos en Tijuana, las alternativas basadas en el enfoque "progresivista" de mejoras y ampliaciones de las viviendas por etapas, resulta pertinente para gran parte de las familias que ya cuentan con un terreno propio y algún tipo de construcción; también, para quienes requieren de una vivienda nueva la provisión de lotes con servicios o con pies de casa parecen opciones viables y adecuadas a las capacidades de pago de los hogares y a sus posibilidades de mantener un ritmo de expansión en la construcción acorde a sus necesidades futuras. Considerando la experiencia de esta ciudad fronteriza, buena parte de los beneficiarios de Tu Casa muestran altos niveles de 
satisfacción respecto de los apoyos recibidos, tanto en la modalidad de ampliación como en la de provisión de una UBV.

No obstante lo elemental que pudiera parecer una intervención gubernamental a esta escala, los limitados alcances del programa y el pésimo desempeño mostrado por la instancia ejecutora (INETT) y la entidad supervisora (delegación de la Sedesol) en Tijuana, son sólo una muestra de las grandes restricciones y deficiencias de la política habitacional seguida por el Estado mexicano para atender las necesidades de los hogares en situación de pobreza en México.

Los considerables rezagos en esta materia obligan a todos los órdenes de gobierno, pero principalmente a la Federación, a poner en marcha medidas tendientes a reforzar y mejorar la intervención de este programa en al menos cuatro aspectos: i) incrementar la cobertura y el número de acciones en las diversas modalidades indicadas en el diseño del programa a partir de diagnósticos realistas sobre las necesidades habitacionales en contextos sociales específicos; ii) alentar la participación de las instancias ejecutoras e incentivar su buen desempeño; iii) mejorar la calidad de los materiales de construcción y con ello el procedimiento de edificación de cuartos y viviendas; y de una manera muy importante, iv) coordinar esfuerzos para canalizar una mayor cantidad de recursos a estas acciones, así como para ampliar los beneficios de otros programas que inciden directamente sobre la problemática habitacional, como los de servicios públicos, regularización y desarrollo comunitario.

\section{Bibliografía}

Coplamar (1986), Necesidades esenciales en México. 3. Vivienda, Siglo XXI, México.

Diario Oficial de la Federación (2002), "Acuerdo por el que se modifican las Reglas de Operación del Programa de Ahorro, Subsidio y Crédito para la Vivienda Progresiva Vivah, para el ejercicio fiscal 2002", 15 de marzo. (2003, 2004 y 2006), “Acuerdos por los que se modifican las Reglas de Operación del Programa de Ahorro, Subsidio y Crédito para la Vivienda Progresiva Tu Casa, para los ejercicios fiscales 
2003, 2004 y 2006", del 27 de febrero de 2003, 7 de mayo de 2004 y 17 de febrero de 2006.

El Colegio de la Frontera Norte (2005), "40 estudios de caso de impacto del programa Hábitat", Apartado metodológico.

Fideicomiso Fondo Nacional de Habitaciones Populares (2003), “Introducción del Programa Institucional de Trabajo 2003".

(2004), “Descripción del Programa de Ahorro y Subsidios para la Vivienda Progresiva", Secretaría de Desarrollo Social.

Gobierno del Estado de Baja California, "Opinión respecto de la Cuenta Pública Anual de Inmobiliaria Estatal de Tijuana-Tecate", Dictamen número 39, Comisión de Fiscalización del Gasto Público.

Hipotecaria Nacional (2005), "Estudio del mercado de vivienda en Baja California", http://www.hipnal.com.mx/not_bc.html.

Inmobiliaria Estatal Tijuana-Tecate (2004), "Cédulas de Información Socioeconómica de los beneficiarios del programa Tu Casa 20022003", Tijuana.

Instituto Nacional de Estadística, Geografía e Informática (2000), Muestra del 10\% del XII Censo General de Población y Vivienda.

Jardón, Ana, (2004), La política habitacional en México, el impacto social del programa Tu Casa en Tijuana, B.C, 2002-2003, tesis de maestría, El Colegio de la Frontera Norte.

Little, Steve (2007), "Porque no hay de otra: la elocuencia de San Cristóbal y Tijuana frente a la vivienda inadecuada", reporte de investigación, Oficina Regional para América Latina y el Caribe de Hábitat para la Humanidad.

Ordóñez, Gerardo (2002), La política social y el combate a la pobreza en México, UNAM, Sedesol, México.

Schteingart, Martha (1988), "Diez años de programas y políticas de vivienda en México", en Gustavo Garza (comp.), Una década de planeación urbano-regional en México, 1978-1988, manuscrito, El Colegio de México.

(1989), "La investigación sobre vivienda en México (1950-1987)", Ciudades, núm. 3, RNIU.

Secretaría de Desarrollo Social (2003), "Medición del desarrollo: México 2000-2002", México. 
Puebla, Claudia (2003), Del intervencionismo estatal a las estrategias facilitadoras. Cambios en la política de vivienda de México, El Colegio de México.

Villavicencio, Judith (1997), "Vivienda para los más pobres: ¿el olvido?", Ciudades, núm. 36, octubre-diciembre.

Recibido en mayo de 2008

Segunda versión recibida en noviembre de 2008

Aprobado en diciembre de 2008 
